\title{
Protomene: a new carbon allotrope
}

\author{
Francesco Delodovici ${ }^{\mathrm{a}}$, Nicola Manini ${ }^{\mathrm{a}}$, Richard S Wittman ${ }^{\mathrm{b}}$, Daniel S. Choi ${ }^{\mathrm{c}}$, Mohamed \\ Al Fahim ${ }^{\text {, }}$ Larry A Burchfield*d \\ ${ }^{a}$ Dipartimento di Fisica, Università degli Studi di Milano, Via Celoria 16, 20133 Milano, Italy \\ ${ }^{b}$ Pacific Northwest National Laboratory, 902 Battelle Blvd, Richland, WA 99354, USA \\ ${ }^{c}$ Mechanical and Materials Engineering Department, Masdar Institute of Science and Technology, A Part \\ of Khalifa University of Science and Technology, PO Box 54224, Abu Dhabi, UAE \\ ${ }^{d}$ Alfields, Inc. PO Box 3091, Richland, WA 99354, USA
}

\begin{abstract}
We introduce a new carbon allotrope named protomene. Its crystal structure is hexagonal, with a fully-relaxed primitive cell involving 48 atoms. Of these, 12 atoms have the potential to switch hybridization between $\mathrm{sp}^{2}$ and $\mathrm{sp}^{3}$, forming dimers. By means of DFT simulations, we have identified the equilibrium structure of protomene, and estimate that it is $2 \%$ less bound than diamond. We have also estimated the amplitude of its direct band gap to be $3 \mathrm{eV}$, and predicted the $\mathrm{X}$-ray diffraction pattern and phonon modes.
\end{abstract}

\section{Introduction}

Driven by the discovery of some of the most interesting new materials, namely fullerenes, nanotubes and graphene, the quest for new allotropes of carbon has been an increasingly active field of research for several decades [1]. This broad interest is fueled by the wide range of structural and electronic properties of carbon allotropes. Indeed, carbon possesses three energetically competitive different types of orbital hybridization $\left(\mathrm{sp}, \mathrm{sp}^{2}\right.$, and $\left.\mathrm{sp}^{3}\right)$.

\footnotetext{
${ }^{*}$ Corresponding author. Tel: +971 505320 130. E-mail: info@ alfields.co
} 
In turn, this allows carbon atoms to combine with each other in an exceptional number of ways. The $\mathrm{sp}^{3}$ configuration gives rise to three-dimensional networks with insulating properties along with high stiffness, as in cubic and hexagonal diamond [2]. In contrast the $\mathrm{sp}$ (linear) and $\mathrm{sp}^{2}$ (planar) hybridizations can be responsible for flexible structures [3, 4] such as carbyne and graphene, which often come with small electronic interband gaps or even metallic properties. Intermediate hybridizations are quite frequent as well, as in the fullerenes and the nanotubes.

Compared to the wide variety of newly identified structures [5], relatively few breakthrough applications have been realized to date. The most common usage of elementary carbon still remains burning. The quest for new applications continues, focusing on electronics, opto-electronics, quantum computing, coatings. This team has recently brought to light a new stable carbon structure combining $\mathrm{sp}^{2}$ and $\mathrm{sp}^{3}$ hybridizations, which was called novamene [6]. In the present work we introduce a new allotrope, protomene, based on similar structural ideas (developed by the corresponding author of the present paper), which in turn exhibits surprisingly different electronic properties compared to novamene.

\section{The crystal structure}

The structure of protomene shares some general concepts with that of the previously studied novamene [6], but with significant novelties. In protomene, 6 atoms out of 24 (compared to 2 out of 26 in novamene) can adopt a perfectly planar $\mathrm{sp}^{2}$ geometry, from which they can then move out of the plane to build comparably weak bonds with partner atoms in the next vertically stacked lattice cell. Instructed by the novamene example, one can expect this extra bond formation to lower the total energy by approximately $1 \mathrm{eV}$ per bond, and to induce a substantial change in electronic properties. 


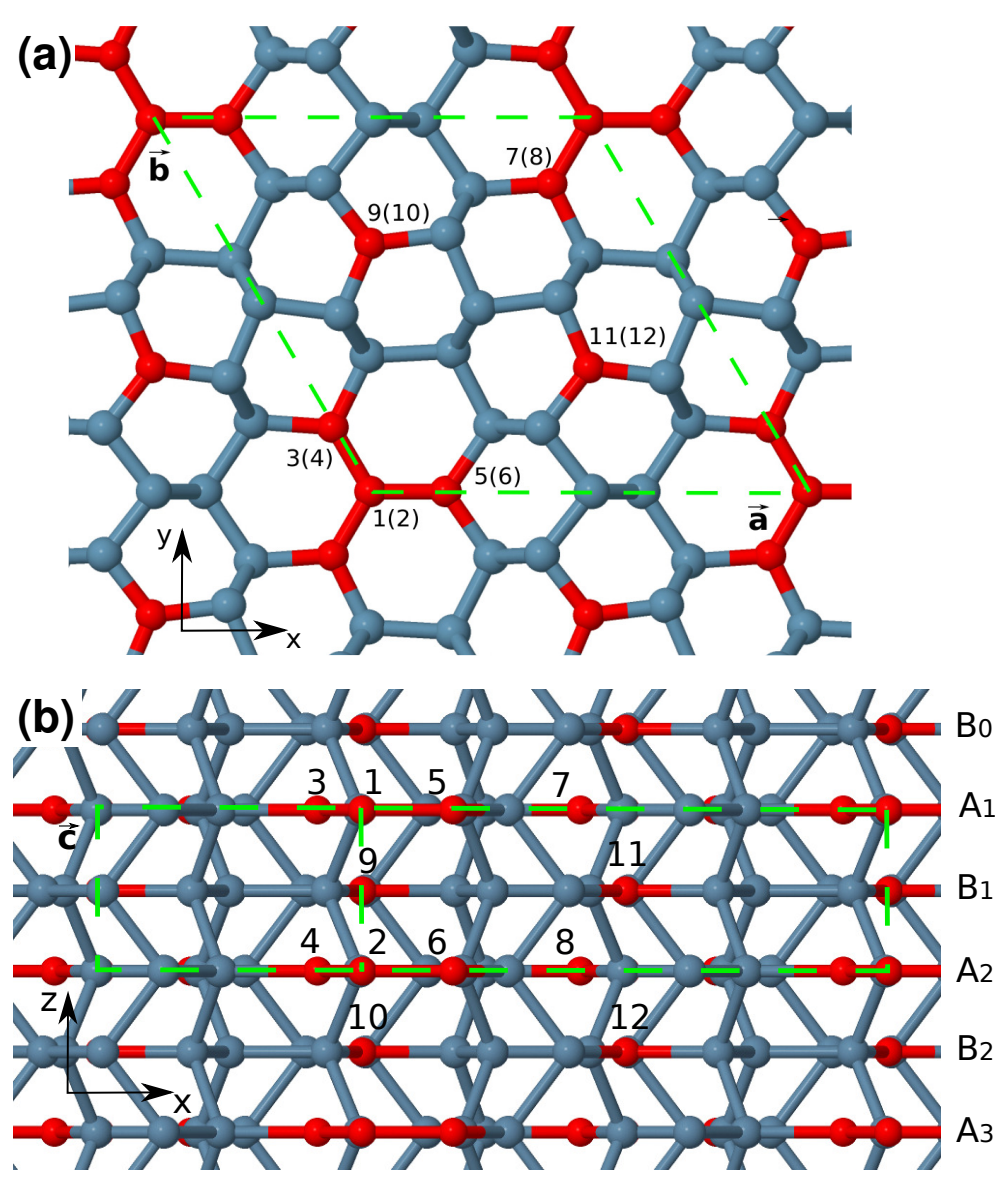

Figure 1: A ball-stick model of the structure of protomene, relaxed in its no-dimer configuration. (a) A view down the threefold-symmetry $\hat{z}$ axis; (b) a side view in the $\hat{y}$ direction. The hexagonal primitive cell is highlighted by green dashed lines and contains 24 atoms. The primitive vectors $\vec{a}, \vec{b}, \vec{c}$ are indicated. The "switching" $\mathrm{sp}^{2}$ carbon atoms are highlighted in red. In this structure these switching atoms are all in their no-dimer (higher-energy) configuration: atoms 1,3,5,7 are equivalent to 2,4,6,8 and they are placed in successive $A$ planes of adjacent cells; atoms 9,11 are equivalent to 10,12 and they occupy successive $B$ planes, which stand at intermediate heights between $A$-type planes. 
Like novamene, the protomene structure has threefold symmetry axes, which are compatible with crystallization in a hexagonal lattice. We constructed a classical ball-and-stick model as an initial conjecture for the structure, and adapted it to a crystal repetition symmetry, as reported in fig. 1. We identified the correct minimum primitive cell before any dimer formation: this primitive cell, containing 24 atoms, is sketched by a dashed line in fig. 1. This figure highlights the $\mathrm{sp}^{2}$ carbon atoms in red: a group of 4 atoms (labeled $1,3,5$, and 7) sits around the corner of the cell, plus two more (labeled 9 and 11) isolated within the cell. Even-number labeled atoms occupy equivalent positions in the next identical layer below, see fig. $1 \mathrm{~b}$. All 18 remaining gray-colored atoms are natively $\mathrm{sp}^{3}$ carbons.

Compared to novamene [6], the main structural novelty of this new allotrope consists in the absence of any benzenic ring. Protomene has only groups of at most four $\mathrm{sp}^{2}$ atoms bonded together. These $\mathrm{sp}^{2}$ atoms have the possibility to form further bonds with similar atoms in adjacent crystalline planes. Since dimer formation usually leads to energy lowering, among all possible realizations of interplane dimers we envisage that the lowestenergy (favored) state will be the one with as many interplane dimers as possible. When any number of dimers is formed, successive $A$ or $B$ planes are not equivalent any more, with the result that the $\hat{z}$-directed $c$ primitive vector doubles in length, implying a primitive-cell doubling, as illustrated in the comparison of figs. 1 and 2. One could conceive extended patterns of dimer formation in the horizontal $x-y$ plane, which would lead to involvement of the $a$ and $b$ primitive vectors as a further cell-sized multiplication. The number of possible patterns of cross-plane dimers would increase exponentially with the number of inequivalent cells involved in the pattern. For simplicity, here we do not consider such 


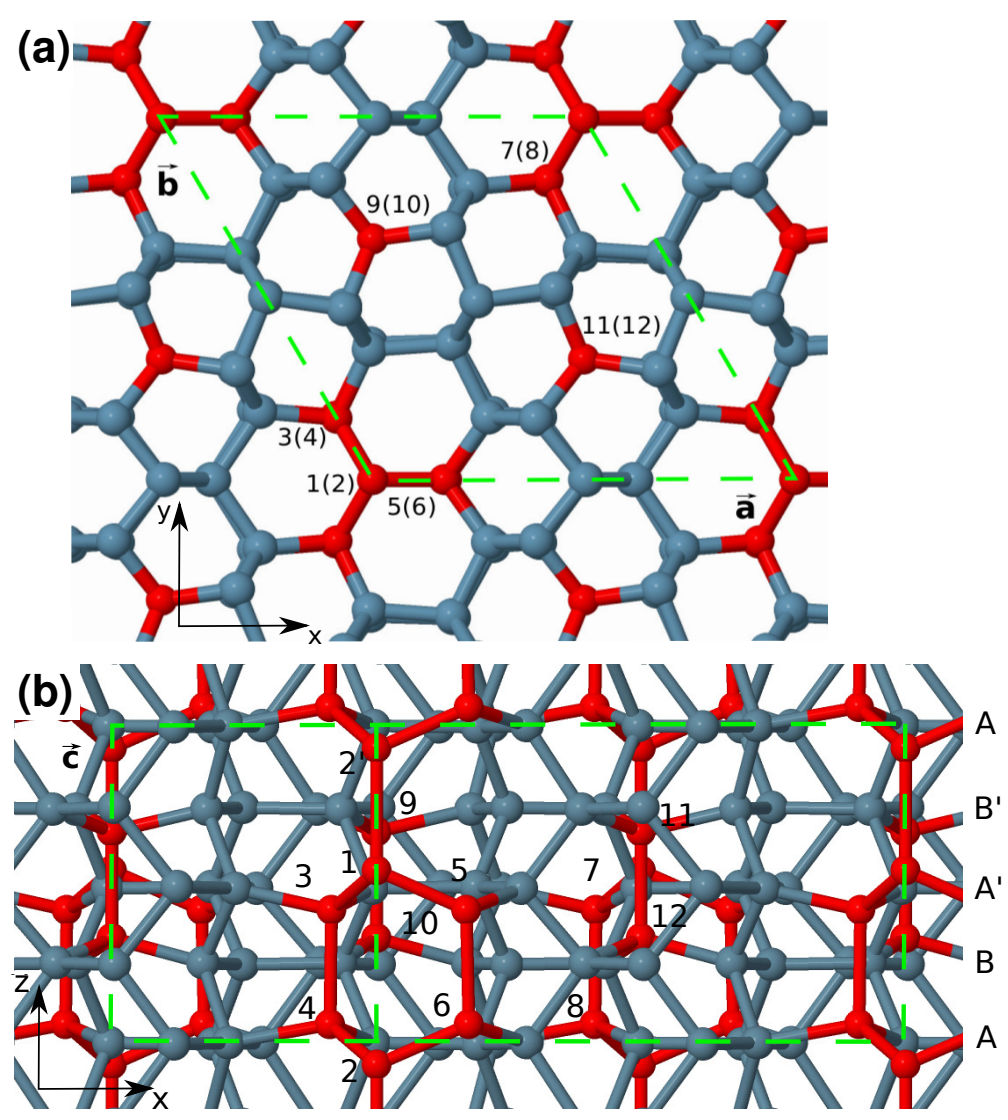

Figure 2: A ball-stick model of the fully relaxed ground-state configuration of protomene, with the energetically most convenient dimerization pattern. (a) top view down the $\hat{z}$ direction; (b) side view in the $\hat{y}$ direction. The $\mathbf{c}$ primitive vector is twice as long compared to the no-dimer configuration because $A^{\prime}$ and $B^{\prime}$ planes are no longer equivalent to $A$ and $B$ planes. The primitive cell is highlighted with a green dashed line.

combinations, instead we only examine all possible dimer patterns within one horizontal primitive cell, combining two 24 -atoms cells stack on top of each other to a full 48 -atoms primitive cell.

First, we observe that the central pairs (9-10 and 11-12) can form independently from each other, since they are structurally rather distant and the distortion induced by the formation of a single dimer remains localized. As a consequence, the configurations, as in fig. 2, with both central dimers formed around plane $A_{2}$ of fig. 1 should have very simi- 
lar cohesive energy compared to the structurally non-equivalent configuration with alternate central dimers, namely one dimer formed around the central $A_{2}$ plane and two others around the $A_{1}$ and $A_{3}$ planes in fig. 1. On the other hand, vertical displacements of the four pairs of corner atoms are strongly correlated with one another, since these atoms are connected by direct in-plane bonds: the formation of one dimer will drive the formation of all three others. Concerning the corner atoms, it turns out that the most stable configuration is the one with alternating dimers: if atoms 1 and 2 bond together across the $B_{1}$ plane, then the neighbouring sp $\mathrm{sp}^{2}$ atoms $(3-6)$ will tend to form bonds across the $B_{0}$ and $B_{2}$ planes of vertically adjacent cells, as shown in fig. 1b. All other possible combinations are likely to be associated with a less favorable cohesive energy. Consider, e.g., the configuration with all corner dimers formed across the same $B$ plane: this is a rather unstable combination, involving three 4-atoms rings with highly deformed bond geometries.

Three possible combinations of the central dimers exist for each arrangement of the corner $\mathrm{sp}^{2}$ carbons. Specifically we have alternatively the formation of: no central dimer, two dimers across the same $B$ plane, or two dimers joining alternating planes. A single dimer would not be stable (as we have verified): the remaining pair of $\mathrm{sp}^{2}$ atoms would move closer too and form a dimer due to the symmetry breaking generated by the formation of the first dimer.

The space group describing the symmetries of different configurations will depend on the dimers pattern: specifically, the 48 -atom-cell structure displayed in fig. 2 is characterized by the space group P-31m (\#157). Given the space-group symmetry one could obtain all (24)48 atomic positions in terms of (7)14 inequivalent ones [7]. 


\begin{tabular}{ccccc}
\hline Atom & $\mathbf{a}_{1}$ & $\mathbf{a}_{2}$ & $\mathbf{a}_{3}$ & $n_{\text {symm }}$ \\
\hline \hline 1 & 0.0000 & 0.0000 & 0.5253 & 1 \\
2 & 0.5048 & 0.0000 & 0.7766 & 3 \\
3 & 0.6667 & 0.3333 & 0.8528 & 2 \\
4 & 0.4721 & 0.1689 & 0.7813 & 6 \\
5 & 0.1746 & 0.0000 & 0.4600 & 3 \\
6 & 0.3759 & 0.1830 & 0.5187 & 6 \\
7 & 0.3825 & 0.3825 & 0.5232 & 3 \\
8 & 0.0000 & 0.0000 & 0.9747 & 1 \\
9 & 0.5057 & 0.0000 & 0.2724 & 3 \\
10 & 0.6667 & 0.3333 & 0.1886 & 2 \\
11 & 0.4817 & 0.1734 & 0.2669 & 6 \\
12 & 0.1693 & 0.0000 & 0.0991 & 3 \\
13 & 0.3603 & 0.1726 & 0.0319 & 6 \\
14 & 0.3764 & 0.3764 & 0.0268 & 3 \\
\hline
\end{tabular}

Table 1: The fractional coordinates of the 14 inequivalent atoms in the lowest-energy dimerized structrure. Columns 2, 3, and 4 report the $u_{j}^{i}$ components of the 14 symmetry-inequivalent carbon atoms at positions $\mathbf{d}_{i}$ in the cell, along the primitive vectors $\mathbf{a}_{j}$ of the hexagonal lattice, according to $\mathbf{d}_{i}=\sum_{j=1}^{3} u_{j}^{i} \mathbf{a}_{j}$. The cell parameters $\left(a=\left|\mathbf{a}_{1}\right|=\left|\mathbf{a}_{2}\right|\right.$ and $\left.c=\left|\mathbf{a}_{3}\right|\right)$ are provided in Table 2 below. Column 5 reports the number $n_{\text {symm }}$ of equivalent atoms generated by applying symmetry operations as indicated in the supplementary material.

\section{Numerical Simulations}

\subsection{Structural properties}

To obtain the equilibrium configurations and the electronic properties of the several competitive structures, we employed density functional theory (DFT) simulations, with a well-established approach $[8,9,10,11,12,13,14,15,16,17,18,19,20]$. We used both local density approximation (LDA) and a general gradient approximation [21, 22] in the Perdew-Burke-Erzoff (PBE) [23] formulation, with ultrasoft pseudo-potentials to account for core electrons [24] as implemented in Quantum Espresso [25]. For both functionals the wave functions are expanded on a plain-waves basis with a kinetic-energy cutoff of $408 \mathrm{eV}$, which guarantees the convergence of the total energy of a simple carbon structure 
(diamond) within $10^{-3} \mathrm{eV}$. We sampled the Brillouin zone of protomene with a $10 \times 10 \times$ 20 Monkhorst-Pack k-point grid [26], and used grids of similar k-space densities (at least 10 points $\times \mathrm{nm}^{3}$ ) for the other calculations to guarantee a similar accuracy of the total energy.

We performed simulations to fully relax the atomic positions $\mathbf{d}_{i}$, also allowing the cell primitive vectors to change to get rid of the overall stress. We started from an initial rough configuration built from the analysis of the structure symmetry as described in Sect. 2 . Atomic relaxation is performed until the following convergence conditions are reached: the total force must be smaller than $4 \mathrm{pN}$ and the total-energy difference between two successive relaxation steps must be smaller than $10^{-3} \mathrm{eV}$. The fully relaxed crystalline structures are provided as Supplementary Content, and Table 1 reports the fractional coordinates of the 14 inequivalent atoms.

Table 2 summarizes the simulation results on the two main relaxed structures of protomene: the no-dimer configuration of fig. 1, and the ground state, namely the configuration with the largest possible number of dimers formed, as displayed in fig. 2. The same quantities for the case of novamene are shown for comparison. The binding energies are calculated as differences relative to diamond. Our DFT simulations result in a LDA binding energy per atom of $-8.7081 \mathrm{eV}$, only $2.2 \%$ less stable than diamond (novamene is $2.6 \%$ less stable than diamond [6]). The PBE functional corresponds to LDA in predicting the protomene ground state to be slightly more stable than novamene. Structures with an intermediate number of dimers, not reported in the table, exhibit intermediate values of the total energy.

We have identified as the ground configuration the one with dimers formed by $9-10$ 


\begin{tabular}{lccccc}
\hline $\begin{array}{l}\text { Structural } \\
\text { parameter }\end{array}$ & $\begin{array}{c}\text { protomene } \\
\text { no-dimer } \\
\text { LDA(PBE) }\end{array}$ & $\begin{array}{c}\text { protomene } \\
\text { ground state } \\
\text { LDA(PBE) }\end{array}$ & $\begin{array}{c}\text { novamene } \\
\text { ground state } \\
\text { LDA(PBE) }\end{array}$ & $\begin{array}{c}\text { diamond } \\
\text { LDA } \\
(\mathrm{PBE})\end{array}$ & $\begin{array}{c}\text { graphite } \\
\text { LDA } \\
(\mathrm{PBE})\end{array}$ \\
\hline \hline$N_{\text {atoms per cell }}$ & 24 & 48 & 52 & 2 & 4 \\
\hline$N_{\text {dimers }}$ corner & - & 4 & - & - & - \\
\hline$N_{\text {dimers }}$ central & - & 2 & 2 & - & - \\
\hline$\Delta E_{\mathrm{b}}$ per atom & 0.2713 & 0.1997 & 0.2272 & 0 & -0.1460 \\
{$[\mathrm{eV}]$} & $(0.1882)$ & $(0.1315)$ & $(0.1350)$ & $(0)$ & $(-0.3176)$ \\
\hline band gap & 0.000 & 3.380 & 0.336 & 4.220 & 0.000 \\
{$[\mathrm{eV}]$} & $(0.000)$ & $(1.274)$ & $(0.371)$ & $(4.445)$ & $(0.000)$ \\
\hline$a$ & 807.2 & 807.4 & 841.9 & 352.3 & 243.3 \\
{$[\mathrm{pm}]$} & $(816.6)$ & $(815.7)$ & $(851.0)$ & $(357.0)$ & $(245.8)$ \\
\hline$c$ & 247.2 & 482.8 & 499.8 & 352.3 & 589.8 \\
{$[\mathrm{pm}]$} & $(250.9)$ & $(497.7)$ & $(509.0)$ & $(357.0)$ & $(644.4)$ \\
\hline density & 3432 & 3512 & 3381 & 3649 & 2639 \\
{$\left[\mathrm{~kg} \mathrm{~m}{ }^{-3}\right]$} & $(3303)$ & $(3338)$ & $(3248)$ & $(3504)$ & $(2366)$ \\
\hline
\end{tabular}

Table 2: The main structural properties of two protomene configurations, the no-dimer one and the dimerized ground state, compared with those of novamene [6], of fcc diamond and of graphite. Binding energy differences $\Delta E_{\mathrm{b}}=E_{\text {protomene }}-E_{\text {diamond }}$, where the reference binding energy of diamond $E_{\mathrm{b}}^{\mathrm{LDA}}=-8.908 \mathrm{eV}$ $\left(E_{\mathrm{b}}^{\mathrm{PBE}}=-8.252 \mathrm{eV}\right)$ is reported per carbon atom, as computed using the LDA or (parenthesized) the PBE exchange and correlation functional. 


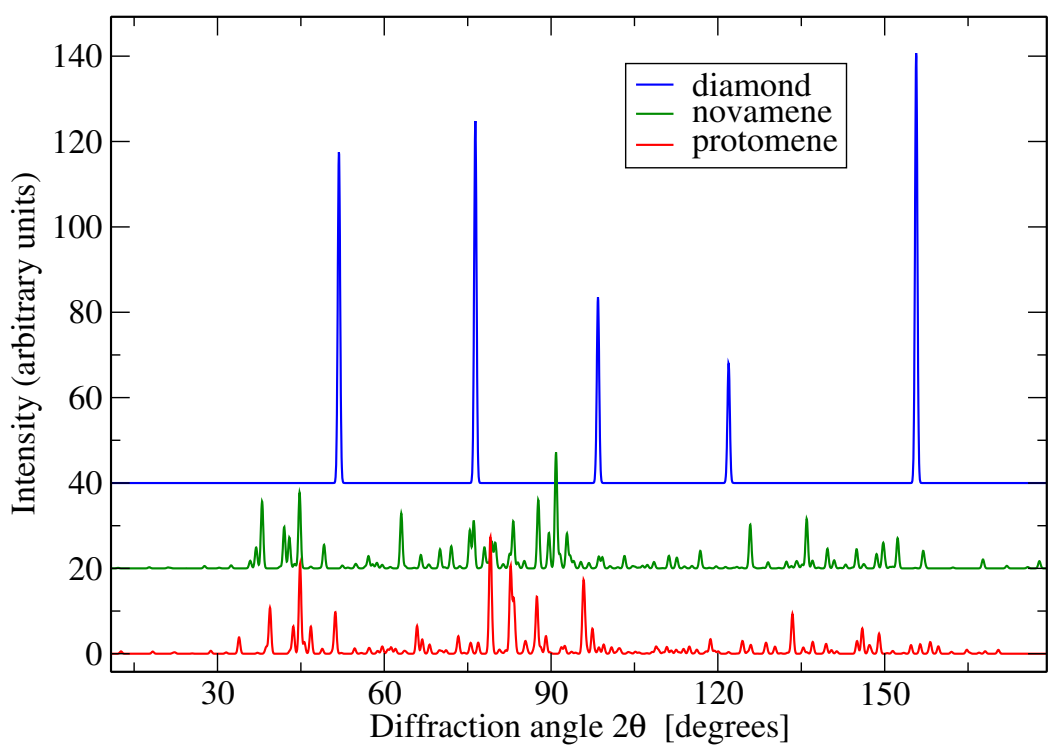

Figure 3: Simulated XRD pattern of protomene (red) in its DFT-LDA ground-state configuration, compared to the diffraction patterns of novamene (green) and diamond (blue), shifted upward for better visibility. The patterns are computed for the standard radiation wavelength $\lambda=154 \mathrm{pm}$ of the $\mathrm{Cu} \mathrm{K}_{\alpha}$ line.

and $11-12$ atoms across the same $A^{\prime}$ plane (as in fig. 2), but the alternative possibility of bonds across alternating $A-A^{\prime}$ planes is essentially degenerate within $1 \mathrm{meV}$ per atom. Although cross-plane bonding occurs independently, the formation of bonds between $B$ and $B^{\prime}$ planes favors the formation of bonds of the other kind by reducing slightly the lattice spacing in the $\hat{z}$ direction. Indeed, as reported in Table 2, $c$ lattice spacing of the ground-state structure is significantly shorter than twice the $c$ spacing of the no-dimer 24-atom-cell structure.

\subsection{Simulated diffraction pattern}

Our DFT simulation provides enough information to evaluate the X-ray diffraction pattern (XRD) of protomene in its ground-state configuration. This pattern is displayed in fig. 3, and compared with the XRD patterns of novamene and diamond. These patterns are obtained for an incident X-ray beam with $\lambda=154 \mathrm{pm}$. The diffracted intensity is 
distributed, as in novamene, among numerous small-intensity peaks, while diamond, with its quite small cell in real space, has widely spaced reciprocal vectors, generating few strong peaks. The predicted pattern of fig. 3 can be used in future experimental attempts to isolate and detect protomene samples.

\subsection{Natural tiling analysis}

It is instructive to examine the structural properties of the protomene allotrope with classification techniques based on a network-topology analysis. To this purpose, we used face symbols to describe the natural tilings of protomene. We refer to articles [27, 28, 29] for the detailed meaning of these topology concepts. In short, a face symbol, characterizing a tile, has the form $\left[A^{\alpha} . B^{\beta} \ldots\right]$. The exponents $\alpha, \beta$ represent the number of faces whose perimeter is a ring with $A, B$ sides. If several tiles are present together, the natural tiling is the sum of all single face symbols. To count the prevalence of each one of the face symbols in the crystal structure, stoichiometric coefficient are introduced. For example: $X\left[A^{\alpha} . B^{\beta}\right]+Y\left[C^{\gamma} . D^{\delta}\right]$ is the natural tiling of a structure with two different tilings appearing in a $X: Y$ ratio.

We made use of the ToposPro software [7] to identify the natural tiling of the protomene ground structure, obtaining:

$$
13\left[6^{3}\right]+3\left[5^{2} \cdot 6^{2}\right]+6\left[6^{4}\right]+3\left[6^{2} \cdot 7^{2}\right]+6\left[5^{2} \cdot 6^{3}\right]+3\left[6^{4} \cdot 7^{2}\right]+3\left[5^{2} \cdot 6^{5}\right]+2\left[6^{9}\right] .
$$

Figure 4 displays the individual tiles forming protomene. Each color corresponds to a different class of tiles. For instance, the thirteen red tiles in figure 4 are part of the $13\left[6^{3}\right]$ cage. Since the single tiling characterizing the diamond structure is $\left[6^{4}\right]$, we deduce that protomene includes $15 \%$ of diamond cages. On the other hand, we exclude the presence of 

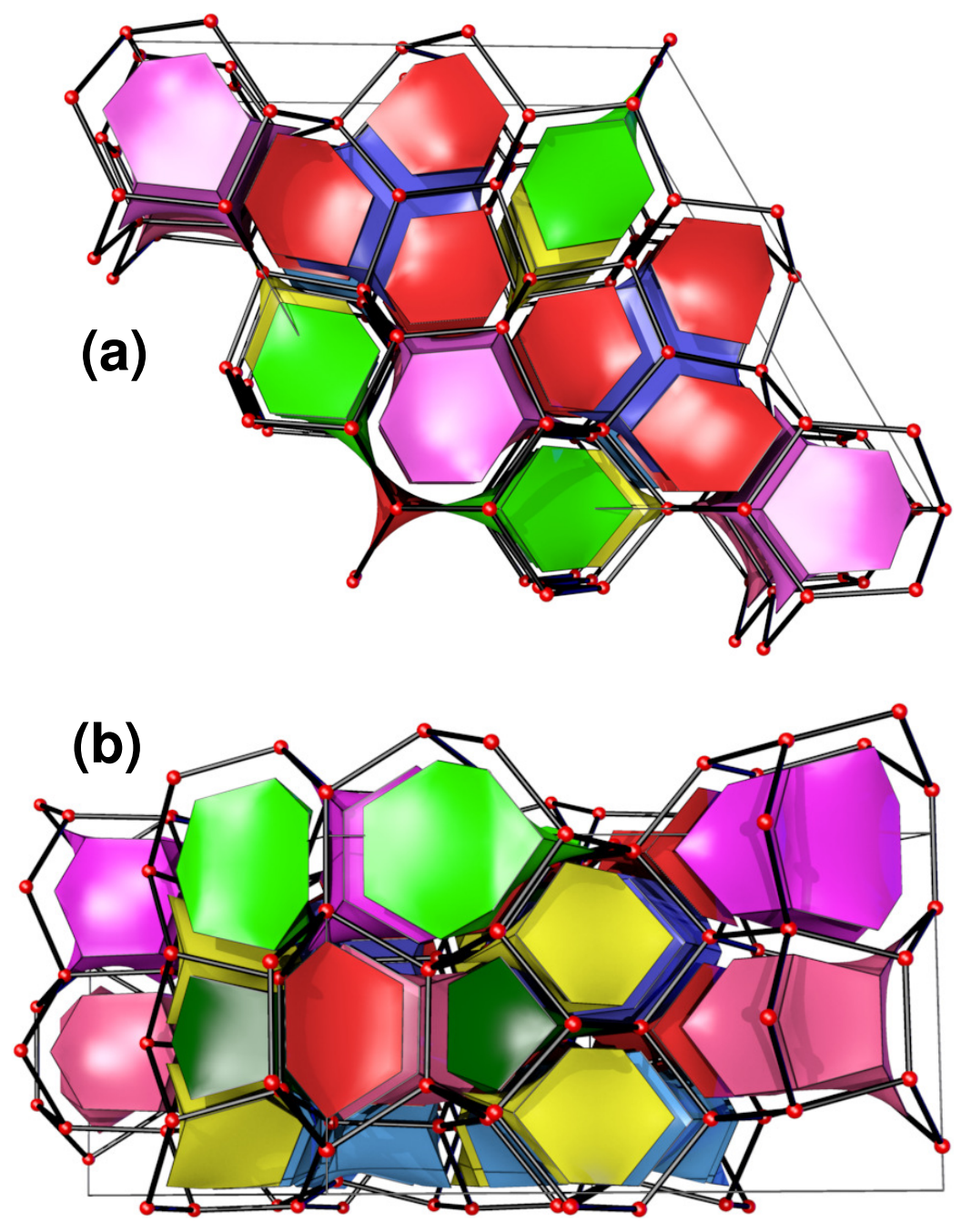

Figure 4: Natural tiling for the protomene structure: (a) top view down from the $\hat{z}$ direction; (b) side view in the $\hat{y}$ direction. Different classes of tiles are labeled by different colors: $13\left[6^{3}\right]$ red, $3\left[5^{2} \cdot 6^{2}\right]$ dark green, $6\left[6^{4}\right]$ yellow, $3\left[6^{2} .7^{2}\right]$ light-green, $6\left[5^{2} \cdot 6^{3}\right]$ light-blue, $3\left[6^{4} .7^{2}\right]$ purple, $3\left[5^{2} \cdot 6^{5}\right]$ pink, $2\left[6^{9}\right]$ blue. 


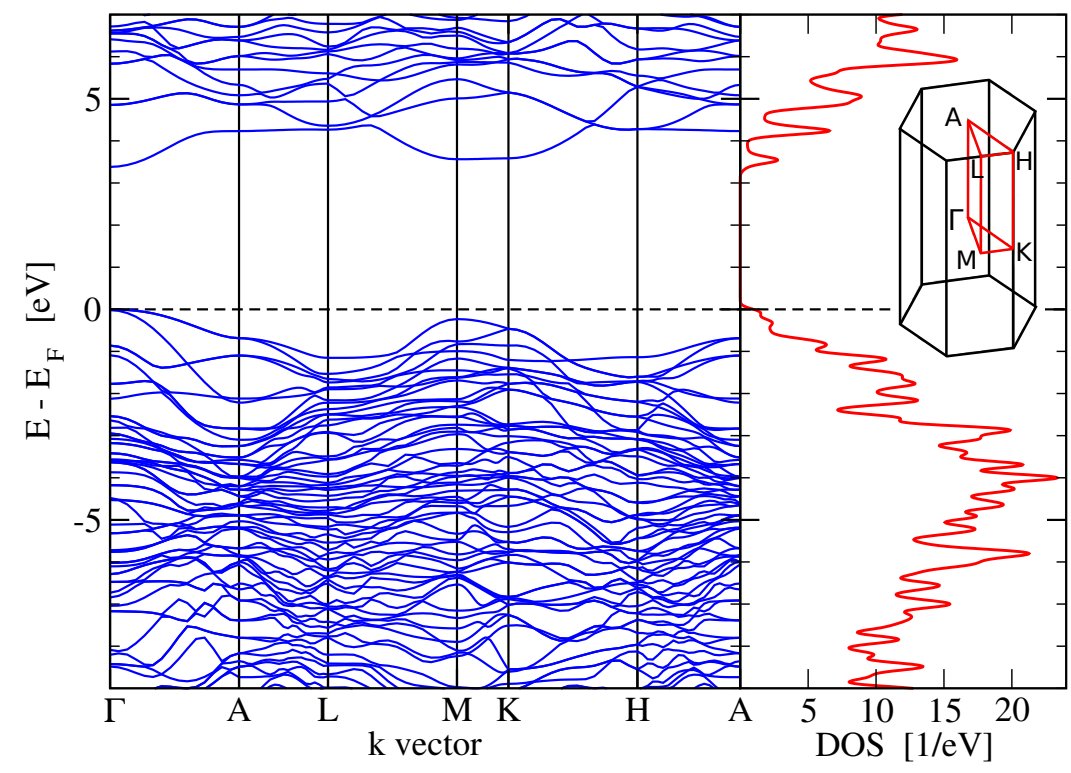

Figure 5: The electronic states of protomene in an energy region around the top of the valence band (dashed line). Left: the DFT-LDA Kohn-Sham band structure along the path in the first Brillouin zone highlighted in the inset at the right side. Right: the density of electronic states of these bands.

regions topologically equivalent to hexagonal diamond (lonsdaleite), classified $\left[6^{3}\right]+\left[6^{5}\right]$, due to the lack of $\left[6^{5}\right]$ in the protomene natural tiling. The obtained topological classification can be useful as an example to search this structure in a database of previously published structures, such as SACADA $[1,30]$. This search is useful in particular to make sure that protomene had not been invented before.

\subsection{Electronic properties}

Figure 5 reports the electron energy bands and the corresponding density of states for the ground-state configuration of protomene. As reported in table 2, the dimer formation involving all initially $\mathrm{sp}^{2}$ atoms produces a gap opening in the band structure at the Fermi level. The band structure depicted in fig. 5 predicts that protomene should be a widebandgap insulator. The DFT-LDA gap amplitude is estimated at $3.38 \mathrm{eV}$. However the 
DFT-PBE simulation estimates the same gap at less than half this energy. The reason for this difference lays in the well-known tendency of DFT-LDA to overbind: the LDA computed interplane dimers are probably shorter and more strongly bonded than in reality, thus generating a larger bonding-antibonding separation, which reflects in the protomene band gap. On the other hand, the DFT-LDA method has a well established reputation for underestimating the band gap. The PBE functional often captures the electronic band gap more accurately than LDA, but its tendency to underbind determines a quite small bonding-antibonding splitting associated with weaker interplane dimerization. As a result, a gap estimation in the $3 \mathrm{eV}$ region is most likely accurate.

The most interesting feature of the protomene band gap is it being a direct gap, with a minimum width at $\Gamma$. Despite the discussed uncertainty about the precise amplitude of the protomene gap, it is significantly larger than in novamene $(0.3-0.4 \mathrm{eV})$, but clearly smaller than in diamond (which however has an indirect gap).

The density of band states reported in fig. 5 shows that, despite the rather flat nature of certain regions of the bands, the overall density of states is fairly smooth. The gap between the valence band and the conduction band is the only gap in this energy region, and the density of states changes from relatively small near the gap itself to rather large a few electron-volts away from it, in both the valence and the conduction regions.

\section{The vibrational spectrum}

In order to simulate the vibrational properties of protomene, we make use of densityfunctional perturbation theory, as implemented in Quantum Espresso [25, 31]. We compute the phonons frequencies along the same $k$-points path as for the electron energy bands.

Figure 6 displays the phonon frequencies along this path in $k$-space. The acoustic 


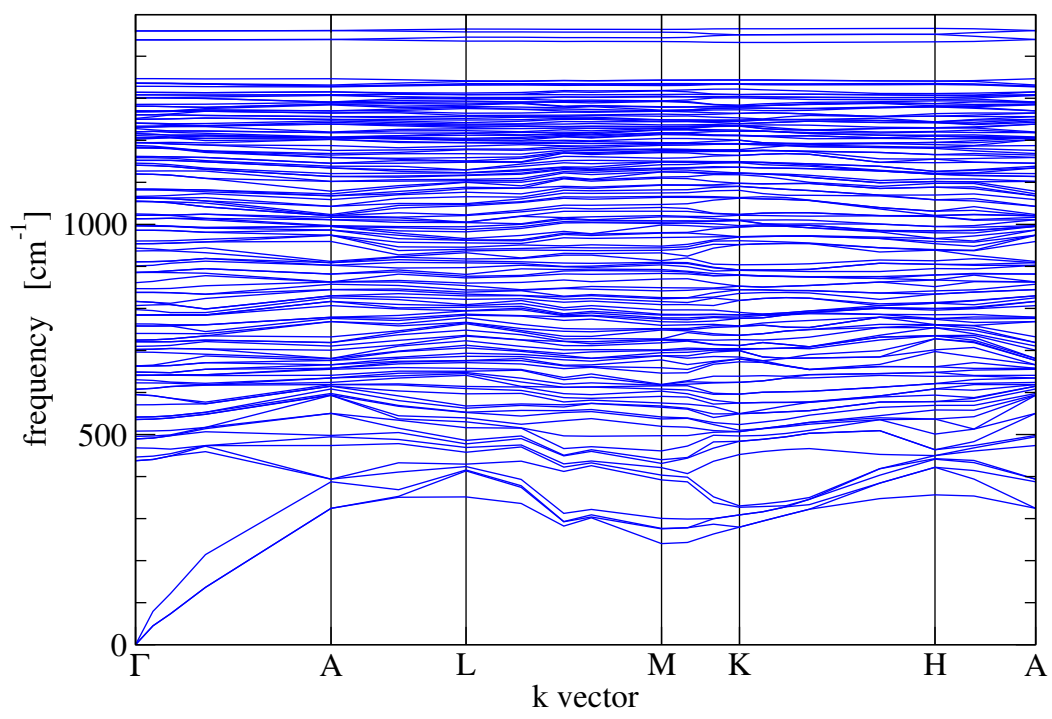

Figure 6: Phonon dispersion computed along the same Brillouin-zone path as drawn for the electron bands in fig. 5.

branches are clearly visible as they depart from zero frequency at $\Gamma$; the lower (transverse) acoustic branch is twofold degenerate along the $\Gamma-\mathrm{A}$ high-symmetry line. At higher frequency, mostly in the $400-1400 \mathrm{~cm}^{-1}$ range, the numerous optical phonon branches cluster and intersect with each other. Near $1500 \mathrm{~cm}^{-1}$ and separated from the great majority of phonon branches by a small gap $\left(\sim 90 \mathrm{~cm}^{-1}\right)$, we find two optical phonons, each of them twofold-degenerate along the $\Gamma-\mathrm{A}$ line. We analyze in particular the atomic displacement corresponding to these optical modes at $\Gamma$ : they are mainly stretching modes of the short nearly-horizontal bonds connecting the atoms labeled 9-11 forming the central dimers with one of those surrounding them. This distinctive spectral feature may provide a useful signature for the experimental identification of the protomene structure. Due to the comparably large number of atoms per cell, the computed vibrational spectrum is consistent with a quite congested vibrational spectrum. Overall, the Raman spectrum will 
probably turn out not too much different from the "D band" of amorphous carbon [32].

\section{Discussion and conclusions}

Protomene qualifies as a new potentially-useful direct-gap semiconductor. The energy band gap (see table 2 ) is very close to that of gallium nitrides $(\mathrm{GaN})$ which is approximately $3.4 \mathrm{eV}$ at room temperature [33]. As a consequence, protomene possesses similar semiconducting properties to $\mathrm{GaN}$, which may enable it to have applications for highpower and/or high-frequency electronic devices with large breakdown voltages. Considering that it is a binary system, the control of $\mathrm{GaN}$ composition is challenging during the crystal growth, whereas protomene is a single-element carbon allotrope and defect control might be easier than for GaN. Due to the gap amplitude near the blue end of the visible spectrum, protomene may find applications in opto-electronic components, e.g. to blue- or UV-light generating light emitting diodes (LED), or as a UV filter in optics. Furthermore, the well-defined direct gap suggests that protomene may have more application in semiconductors devices than carbon nanotubes (CNT) and graphene in terms of energy band gap. Indeed, one obstacle to overcome in the fabrication of CNT is controlling whether the CNT is metallic and semiconducting. Protomene instead is predicted to be semiconducting up to a transition temperature.

Thermal expansion in protomene is likely to play against the interplane bonding. Therefore, as temperature is raised, a structural phase transition is likely occur from the lowtemperature semiconduting 48 -atoms cell structure of fig. 2 to the high-temperature metallic phase characterized by the 24 -atoms cell structure of fig. 1 . As this transition is approached, the band gap would close rapidly, much faster than the slow decay, also associated to thermal expansion, in diamond and silicon. Accordingly, this phase transition 
would provide a sensitive temperature-controlled optical filter. Additionally the eventual transition to the high-temperature no-dimers metallic phase of protomene, has potential temperature-controlled optical- and electric-switching applications. Moreover, given the

increasing number of $\mathrm{sp}^{2}$ bonds, this phase transition is likely to undermine the hardness of protomene in its insulating phase. Therefore, protomene is expected to exhibit a significant lowering in hardness as the temperature is raised. As a rough estimate, graphite and diamond are can be taken as lower and upper bounds for the hardness of the high- and low-temperature phases of protomene respectively.

In the future, we consider building on the know-how acquired in the present piece of research to extend the quest for new carbon allotropes, for example considering the possibility of interconnecting $\mathrm{sp}$ chains with $\mathrm{sp}^{3}$ scaffolds.

\section{Acknowledgments}

We are grateful to Davide M. Proserpio for useful discussion and suggestions.

\section{Appendix A. Supplementary data}

Supplementary data related to this article can be found at .... Supplementary data consist in two files: protomene.24.cif and protomene.48.cif, which, in standard crystallographic format, describe quantitatively the no-dimer structure of fig. 1 and the DFT-LDA relaxed ground-state structure of fig. 2, respectively.

\section{References}

[1] R. Hoffmann, A. A. Kabanov, A. A. Golov, D. M. Proserpio, Homo citans and carbon allotropes: for an ethics of citation, Angew. Chem. Int. Ed. 55 (37) (2016) 1096210976. 
[2] M. R. Salehpour, S. Satpathy, Comparison of electron bands of hexagonal and cubic diamond, Phys. Rev. B 41 (5) (1990) 3048-3052. doi:10.1103/PhysRevB.41.3048.

[3] A. H. Castro Neto, F. Guinea, N. M. R. Peres, K. S. Novoselov, A. K. Geim, The electronic properties of graphene, Rev. Mod. Phys. 81 (1) (2009) 109-162. doi:10.1103/RevModPhys.81.109.

[4] A. Eatemadi, H. Daraee, H. Karimkhanloo, M. Kouhi, N. Zarghami, A. Akbarzadeh, M. Abasi, Y. Hanifehpour, S. W. Joo, Carbon nanotubes: properties, synthesis, purification, and medical applications, Nanoscale Res. Lett. 9 (1) (2014) 393-406. doi:10.1186/1556-276X-9-393.

[5] V. L. Deringer, G. Csányi, D. M. Proserpio, Extracting crystal chemistry from amorphous carbon structures, ChemPhysChem 18 (8) (2017) 873-877. doi:10.1002/cphc.201700151.

[6] L. A. Burchfield, M. A. Fahim, R. S. Wittman, F. Delodovici, N. Manini, Novamene: A new class of carbon allotropes, Heliyon 3 (2) (2017) e00242-e00257. doi:http://doi.org/10.1016/j.heliyon.2017.e00242.

[7] V. A. Blatov, A. P. Shevchenko, D. M. Proserpio, Applied topological analysis of crystal structures with the program package topospro, Crys. Growth \& Des. 14 (7) (2014) 3576-3586. doi:10.1021/cg500498k.

[8] T. Kawai, Y. Miyamoto, O. Sugino, Y. Koga, Graphitic ribbons without hydrogentermination: Electronic structures and stabilities, Phys. Rev. B 62 (24) (2000) R16349-R16352. doi:10.1103/PhysRevB.62.R16349. 
[9] M. Lüders, A. Bordoni, N. Manini, A. Dal Corso, M. Fabrizio, E. Tosatti, Coulomb couplings in positively charged fullerene, Philos. Mag. Part B 82 (15) (2002) 16111647. doi:10.1080/13642810208220729.

[10] Y. Liu, R. Jones, X. Zhao, Y. Ando, Carbon species confined inside carbon nanotubes: a density functional study, Phys. Rev. B 68 (12) (2003) 125-413.

[11] T. Wassmann, A. P. Seitsonen, A. M. Saitta, M. Lazzeri, F. Mauri, Structure, stability, edge states, and aromaticity of graphene ribbons, Phys. Rev. Lett. 101 (9) (2008) $096-402$.

[12] S. Okada, Energetics of nanoscale graphene ribbons: Edge geometries and electronic structures, Phys. Rev. B 77 (2008) 041408-041412. doi:10.1103/PhysRevB.77.041408.

[13] L. Ravagnan, N. Manini, E. Cinquanta, G. Onida, D. Sangalli, C. Motta, M. Devetta, A. Bordoni, P. Piseri, P. Milani, Effect of axial torsion on sp carbon atomic wires, Phys. Rev. Lett. 102 (24) (2009) 245502-245506. doi:10.1103/PhysRevLett.102.245502.

[14] M. Cazzaniga, L. Caramella, N. Manini, G. Onida, Ab initio intraband contributions to the optical properties of metals, Phys. Rev. B 82 (2010) 035104-035111. doi:10.1103/PhysRevB.82.035104.

[15] E. Hobi Jr., R. Pontes, A. Fazzio, A. da Silva, Formation of atomic carbon chains from graphene nanoribbons, Phys. Rev. B 81 (2010) 201406-201410. doi:10.1103/PhysRevB.81.201406. 
[16] I. E. Castelli, P. Salvestrini, N. Manini, Mechanical properties of carbynes investigated by ab initio total-energy calculations, Phys. Rev. B 85 (21) (2012) 214-110.

[17] Z. Zanolli, G. Onida, J.-C. Charlier, Quantum spin transport in carbon chains, ACS Nano 4 (9) (2010) 5174-5180. doi:10.1021/nn100712q.

[18] I. E. Castelli, N. Ferri, G. Onida, N. Manini, Carbon sp chains in graphene nanoholes, J. Phys. Condens. Matter 24 (10) (2012) 104019-104037. doi:10.1088/09538984/24/10/104019.

[19] E. Cinquanta, N. Manini, L. Ravagnan, L. Caramella, G. Onida, P. Milani, P. Rudolf, Oxidation of carbynes: Signatures in infrared spectra, J. Chem. Phys. 140 (24) (2014) 244708-244714.

[20] M. Esser, A. A. Esser, D. M. Proserpio, R. Dronskowski, Bonding analyses of unconventional carbon allotropes, Carbon 121 (2017) 154 - 162. doi:https://doi.org/10.1016/j.carbon.2017.05.062.

[21] A. D. Becke, Density-functional exchange-energy approximation with correct asymptotic behavior, Phys. Rev. A 38 (1988) 3098-3100. doi:10.1103/PhysRevA.38.3098.

[22] J. P. Perdew, J. A. Chevary, S. H. Vosko, K. A. Jackson, M. R. Pederson, D. J. Singh, C. Fiolhais, Atoms, molecules, solids, and surfaces: Applications of the generalized gradient approximation for exchange and correlation, Phys. Rev. B 46 (11) (1992) 6671-6687. doi:10.1103/PhysRevB.46.6671. 
[23] J. P. Perdew, K. Burke, M. Ernzerhof, Generalized gradient approximation made simple, Phys. Rev. Lett. 77 (18) (1996) 3865-3868. doi:10.1103/PhysRevLett.77.3865.

[24] F. Favot, A. Dal Corso, Phonon dispersions: Performance of the generalized gradient approximation, Phys. Rev. B 60 (1999) 11427-11431. doi:10.1103/PhysRevB.60.11427.

[25] P. Giannozzi, S. Baroni, N. Bonini, M. Calandra, R. Car, C. C. et al., Quantum espresso: a modular and open-source software project for quantum simulations of materials, J. Phys. Condens. Matter 21 (39) (2009) 395502-395521.

[26] H. J. Monkhorst, J. D. Pack, Special points for brillouin-zone integrations, Phys. Rev. B 13 (1976) 5188-5192. doi:10.1103/PhysRevB.13.5188.

[27] V. A. Blatov, M. O’Keeffe, D. M. Proserpio, Vertex-, face-, point-, schläfli-, and delaney-symbols in nets, polyhedra and tilings: recommended terminology, CrystEngComm 12 (1) (2010) 44-48. doi:10.1039/B910671E.

[28] N. A. Anurova, V. A. Blatov, G. D. Ilyushin, D. M. Proserpio, Natural tilings for zeolite-type frameworks, J. Phys. Chem. C 114 (22) (2010) 10160-10170. doi:10.1021/jp1030027.

[29] I. A. Baburin, D. M. Proserpio, V. A. Saleev, A. V. Shipilova, From zeolite nets to sp 3 carbon allotropes: a topology-based multiscale theoretical study, Phys. Chem. Chem. Phys. 17 (2) (2015) 1332-1338. doi:10.1039/C4CP04569F.

[30] D. M. Proserpio, A. A. Golov, A. A. Kabanov, the "Samara Carbon Allotrope Database" - SACADA, http: // sacada.sctms.ru/, 2012. 
[31] S. Baroni, S. de Gironcoli, A. Dal Corso, P. Giannozzi, Phonons and related crystal properties from density-functional perturbation theory, Rev. Mod. Phys. 73 (2001) 515-562. doi:10.1103/RevModPhys.73.515.

[32] M. Marton, M. Vojs, E. Zdravecká, M. Himmerlich, T. Haensel, S. Krischok, M. Kotlár, P. Michniak, M. Veselý, R. Redhammer, Raman spectroscopy of amorphous carbon prepared by pulsed arc discharge in various gas mixtures, J. Spectrosc. 2013 (2012) 467079-467086.

[33] K. Miwa, A. Fukumoto, First-principles calculation of the structural, electronic, and vibrational properties of gallium nitride and aluminum nitride, Phys. Rev. B 48 (1993) 7897-7902. doi:10.1103/PhysRevB.48.7897. 